\title{
Observations of Molecules in Comets
}

\author{
D. Despois ${ }^{1}$, N. Biver ${ }^{2}$, D. Bockelée-Morvan ${ }^{2}$, and J. Crovisier ${ }^{2}$ \\ ${ }^{1}$ Obs. de Bordeaux, OASU/L3AB, BP89, F-33270 Floirac, France \\ email: despois@obs.u-bordeaux1.fr \\ ${ }^{2}$ LESIA, Obs. de Paris, 5 pl. Janssen, F-92195 Meudon, France
}

\begin{abstract}
Comets are among the most primitive bodies of the solar system, and their chemical composition is rich in information on the protosolar nebula and its possible connection with interstellar cloud chemistry. Comets are also a source of light atoms and, possibly, prebiotic organic molecules for the early Earth. We know better and better the abundances of cometary volatiles through spectroscopy, mainly at infrared and radio wavelengths. Another crucial component of cometary matter - organic refractories - is still poorly characterized, however.

We summarize here the $\sim 30$ abundances and $\sim 20$ upper limits obtained on cometary volatiles and highlight a few species and problems: ethylene glycol, $\mathrm{NS}, \mathrm{HNC} / \mathrm{HCN},{ }^{14} \mathrm{~N} /{ }^{15} \mathrm{~N}$, the origin of $\mathrm{CN}, \mathrm{CS}_{2}, \mathrm{PAHs}$, and $\mathrm{H}_{2} \mathrm{O}$. Comet-to-comet variations and comet internal heliocentric variations can now be studied, and cometary comas can be mapped with a variety of techniques. We list a number of temperature indicators, for they can help understand the relation between IS and cometary matters, which present both a global similarity but also marked differences, such as the high ethylene glycol content of comets.

We conclude by outlining a few key problems to be addressed by future ground-based and space instruments or by cometary sample analysis. For many species, we stress that laboratory data are missing on spectroscopy, photodissociation and collisions with $\mathrm{H}_{2} \mathrm{O}$.
\end{abstract}

Keywords. astrochemistry — comet: general — planetary systems: protoplanetary disks — solar system: formation

\section{Introduction}

On the 4 th of July, 2005, comets were again a hot topic, as the Deep Impact projectile hit the $5 \times 7 \mathrm{~km}$ nucleus of comet $9 \mathrm{P} /$ Tempel 1 . The experiment has brought a wealth of information on the physics of the nucleus, surface details and strength of cometary matter (A'Hearn et al. 2005); it may also have provided new insights in the chemical composition: beside known species, Spitzer IR observations (Lisse et al. 2005) may have detected $\mathrm{Al}_{2} \mathrm{O}_{3}$, and PAHs (already tentatively detected in $1 \mathrm{P} / \mathrm{Halley}$, see below). More results are given in Meech et al. (2005) and Keller et al. (2005).

Comets are the most pristine bodies in the solar system. Their astrochemical interest is at least fourfold: (i) they hold a wealth of information on the physics and chemistry of the protosolar nebula (PSN), (ii) they offer a link, at least partial, to the chemistry of the parent cloud from which the solar system has formed, (iii) they are, through violent impacts as well as through smooth delivery of dust particles (meteors, micrometeorites, IDPs) one source of carbon and prebiotic molecules for the early Earth; (iv) finally, as all astronomical objects presented in this Symposium, they are a remarkable laboratory for molecular physics and chemistry. Kinetic temperatures in the coma first decrease from $\sim 200 \mathrm{~K}$ (water ice sublimation) to a few $10 \mathrm{~K}$ or even less, due to adiabatic expansion, then warm up again due to heating by photodissociation. Rotational populations, first at LTE, progressively decouple from the gas translational energy, due to the rapid decrease in density and collisions far from the nucleus. Solar radiation then takes over as the main 
source of excitation. For vibrational and electronic states, even very close to the nucleus solar excitation dominates, provided the coma is not optically thick. The coma density can be as high as $10^{12} \mathrm{~cm}^{-3}$ close to the surface of the nucleus, and decreases rapidly to the interplanetary level $\left(\sim 1 \mathrm{~cm}^{-3}\right)$. Gas expansion velocities are on the order of $1 \mathrm{~km} \mathrm{~s}^{-1}$. The dominant coma species is usually water, except far $(>4 \mathrm{AU})$ from the Sun, where CO takes over this role. Ions and radicals are photo-produced which means that ion-molecule and radical reactions are possible; ions finally interact with the solar wind, building up the plasma tail.

The time scales for chemistry are diverse. Icy planetesimals can form over periods as short as $2.5 \times 10^{5} \mathrm{yr}$ according to models, and their formation must take place when a gaseous disk still encircles the Sun, i.e. during the first $\sim 10 \mathrm{Myr}$ of the solar system. Note that some ingredients, especially among dust particles, are prestellar, and thus older. The formation is followed by a 4.5 Gyr mostly dormant phase, in one of the two comet reservoirs: the Oort Cloud or the Kuiper Belt. Despite the generally very low temperature $(<30 \mathrm{~K}$ ?) of the nucleus, it is not certain that such a long period is devoid of chemical transformation (an episode of liquid water is even considered as possible in the core of the largest - a few $100 \mathrm{~km}$ - KB objects). The active phase during which the coma is observed typically lasts months, whereas the lifetimes of sublimated molecules range from $10^{3}$ to $10^{6} \mathrm{~s}$ (at $\left.1 \mathrm{AU}\right)$.

For physicists and chemists unfamiliar with comet science, a general introduction is given in Despois \& Cottin (2006), and Crovisier (2004), with special emphasis on the connection to astrobiology. A very recent, deep and complete summary on cometary science is provided by the book Comets II (eds. Festou et al. 2004), where the chapters by Bockelée-Morvan et al. (parent species) and Feldman et al. (secondary species) are the most directly relevant to astrochemistry. The possible connection between ISM and comets is addressed in detail by Irvine et al. (2000) and Ehrenfreund et al. (2004). We will discuss in the following mainly cometary volatiles; when there is no reference to other authors, the original data are from Bockelée-Morvan et al. (2000) and Crovisier et al. (2004a,b; detections and upper limits in comet Hale-Bopp), and Biver et al. (2002a, 2005a) and Crovisier et al. (2005; inter and intra comet variations).

\section{The Composition of Cometary Volatiles}

Comets, if the coma composition reflects that of the nucleus, are made of roughly equal masses of dust and ices (within a factor of 10, depending on the comet). Dust itself is half rocky material (e.g., silicates), half organic refractories ("CHON" particles). We will mainly discuss here the volatile composition. One should, however, keep in mind that: (i) many complex molecules may be hidden in the organic refractories, and are not yet accessible to precise identification; (ii) macromolecular carbon (e.g., heterogeneous copolymers, $c f$. Fomenkova 1999) may be the dominant form of carbon in these refractories as it is in meteorites (Binet et al. 2004).

Figure 1 gives "typical" cometary volatile abundances, together with the range observed in various comets, and the number of comets observed/detected in a given species. "Typical abundances" means in practice that most coma abundances are from C/1995 O1 (Hale-Bopp) observed near $r_{h}=1 \mathrm{AU}$; however, a few species have been measured under more favorable circumstances $\left(\mathrm{CO}_{2}\right)$ or only $\left(\mathrm{S}_{2}, \mathrm{C}_{4} \mathrm{H}_{2}\right)$ in other comets . The comet-tocomet dispersion, comet internal variation, and coma vs. nucleus abundance discussions (including differential sublimation and coma chemistry) should not be forgotten when using these data. 


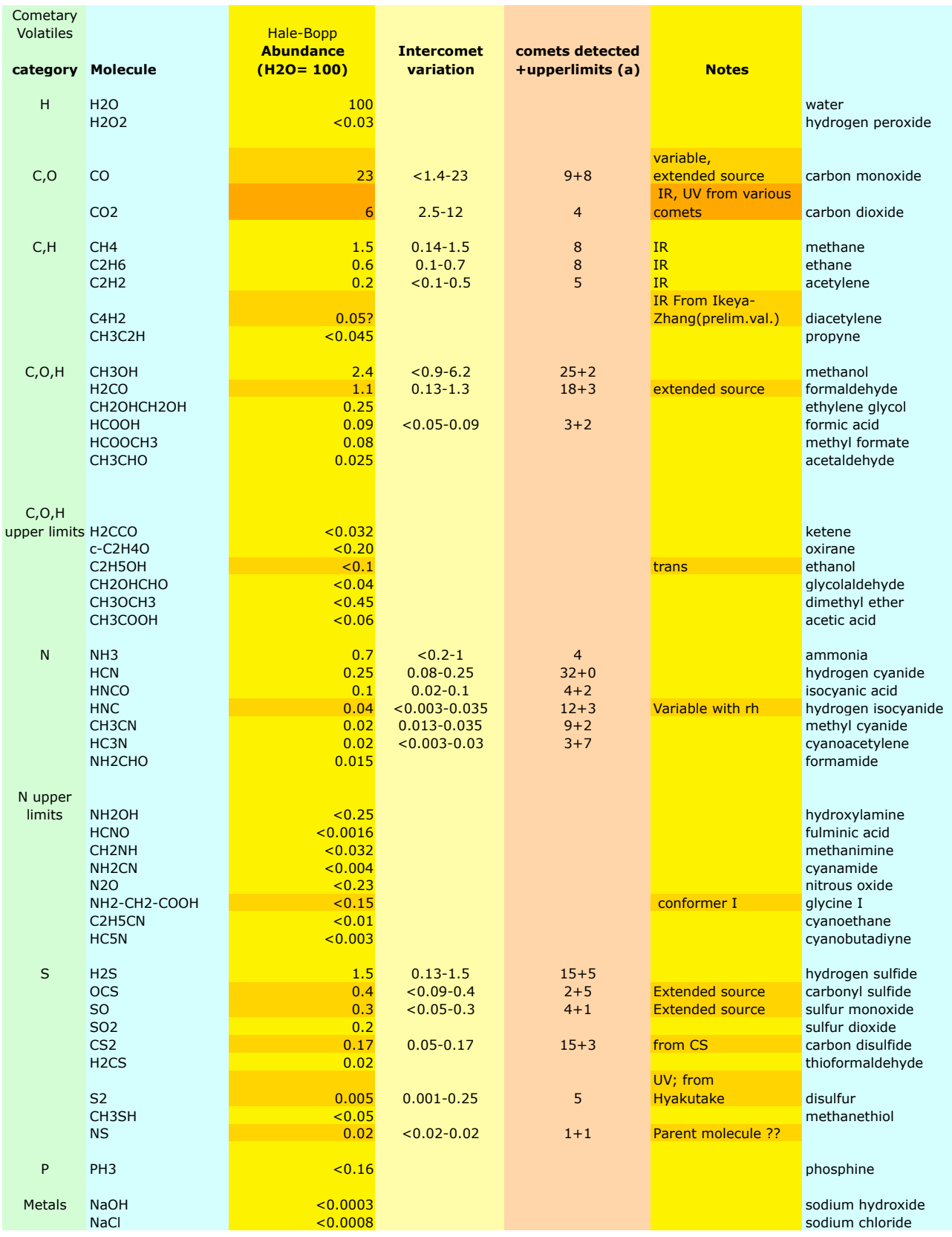

Figure 1. Cometary volatiles: detections and upper limits. Abundances are in $\%$ by number relative to water. (a) refers to radio data unless otherwise noted.

It is of prime importance, when comparing these abundances to laboratory experiments on interstellar/cometary ice analogs, or to chemical models of the ISM or protosolar disk/nebula, to take into account both detections and upper limits. One should especially keep in mind that the modelling used to derive upper limits is less elaborate than that used for the major species, e.g., $\mathrm{H}_{2} \mathrm{O}$ or HCN: LTE is typically assumed, and 
photodissociation rates are in several cases only educated guesses - due to the lack of precise laboratory data. However, radio single dish observations of comet Hale-Bopp by Crovisier et al. (2004a) did not suffer too much from these approximations, as the inner coma inside the beam was dense and little affected by photodissociation.

\section{A Few Highlights}

\subsection{Ethylene Glycol $\mathrm{CH}_{2} \mathrm{OHCH}_{2} \mathrm{OH}$}

Ethylene glycol (EG) has been securely identified in comet Hale-Bopp from IRAM 30m, $\mathrm{PdBI}$ and CSO observations (Crovisier et al. 2004b). Ten transitions with $\mathrm{S} / \mathrm{N}$ of 3 to 10 , spanning the range 20 to $100 \mathrm{~K}$ in upper level energy, were detected. The derived abundance is $0.29 \%$ if one takes the rotational temperature of $77 \mathrm{~K}$ derived from the rotational plot, or $0.17 \%$ if one adopts the $110 \mathrm{~K}$ average rotational temperature derived from other coma species. The photodissociation rate was assumed to have the value $2.10^{-5} \mathrm{~s}^{-1}$ at $\mathrm{AU}$, as no data were available; but the small size of the beam for these observations makes this uncertainty not too critical.

Ethylene glycol is a known IS species, observed for the first time by Hollis et al. (2002 and this volume) in Sgr B2, but some striking differences are apparent when comparisons are made with other molecules. The $\mathrm{EG} / \mathrm{CH}_{3} \mathrm{OH}$ ratio is $\sim 0.1$ in comets, whereas it is $\sim 0.001$ towards Sgr B2; glycolaldehyde, $\mathrm{CH}_{2} \mathrm{OHCHO}$, a molecule very similar to ethylene glycol, has not yet been detected in comets $(x<0.16 \mathrm{EG})$ whereas it has comparable abundances in Sgr B2. More remarkably, ethanol, a simple alcool $\left(\mathrm{CH}_{3} \mathrm{CH}_{2} \mathrm{OH}\right)$ has not yet been detected in comets, while ethylene glycol, a dialcohol, has: $\mathrm{CH}_{3} \mathrm{CH}_{2} \mathrm{OH} / \mathrm{EG}<$ 0.4. All this seems to indicate a specific production mechanism in comets. Considering the high relative abundance of methanol in comets $(2.5 \%$ in Hale-Bopp, up to $6.2 \%$ in other comets) it is tempting to invoke a direct formation from methanol, e.g., by ice irradiation. This has been experimentally performed with success (e.g., Moore, this volume, on $\mathrm{H}_{2} \mathrm{O}-$ $\mathrm{CO}_{2}$ and $\mathrm{H}_{2} \mathrm{O}-\mathrm{CH}_{3} \mathrm{OH}$ ices). One should note, however, that $\mathrm{HCOOH}$ is also produced in large quantities in these experiments, whereas it is even less abundant in comets $(\sim 0.3$ EG) than in Sgr B2 ( EG).

\section{2. $N S$}

The NS radical has been detected in comet Hale-Bopp by Irvine et al. (2000) with the JCMT through its $J=15 / 2-13 / 2 a$ and $f$ transitions, with an abundance $x_{\mathrm{NS}}>0.02 \%$. NS is a well known IS molecule present in both warm and cold clouds. Searches through the $J=11 / 2-9 / 2 e$ and $f$ transitions at $253 \mathrm{GHz}$ in comet Hale-Bopp (Crovisier et al. 2004a) and 153P/Ikeya-Zhang (Biver et al. 2005a) led to upper limits of $0.01 \%$ and $0.02 \%$ respectively, assuming (?) a parent molecule distribution.

The origin of NS is not yet known. Could it be present in the ice? If it is a photodissociation product, which is the parent molecule? $\mathrm{NH}_{4} \mathrm{SH}$ (predicted as a cometary species by Fegley et al. 1993) is a possibility, but its photodissociation behavior is unknown. $\mathrm{NH}_{2} \mathrm{SH}$ is another candidate. No map exists, so we have no constraint on the NS parent lifetime, which also prevents a good estimate of its production rate. The $x_{\mathrm{NS}}>0.02 \%$ abundance in comet Hale-Bopp is a lower limit and assumes a NS photodissociation rate $\beta \sim 0.1-$ $2 \times 10^{-4} \mathrm{~s}^{-1}$. A chemical model has been proposed to produce NS by coma reactions, but it cannot account quantitatively for the NS abundance (Canaves et al. 2002).

\section{3. $H N C$ vs. $H C N$}

The HNC/HCN ratio is seen to decrease in the coma with increasing heliocentric distance $r_{h}$. Irvine et al. (1998) proposed a chemical conversion in the inner coma by ion-molecule reactions and electronic recombination of the molecular ion $\mathrm{HCNH}^{+}$as in cold dark 
clouds. Reactions of HCN with suprathermal hydrogen atoms issued from photodissociation were shown to be more efficient, and capable of quantitatively explaining the comet Hale-Bopp results, but still not the presence of HNC in less dense comas (Rodgers and Charnley 2001, Charnley et al. 2002). To match the HNC/HCN vs. $r_{h}$ data (Biver et al. 2002b, 2005a; Irvine et al. 2003), thermo-desorption from grains is also proposed. Comet Hale-Bopp interferometric observations (Bockelée-Morvan et al. 2005a,b) and new observations (Biver et al. 2005a; Crovisier et al. 2005), which show a plateau in $\mathrm{HNC} / \mathrm{HCN}$ around 0.2 for $r_{h}=0.1-0.5 \mathrm{AU}$, favor thermo-desorption and do not seem to be compatible with HNC production by chemical reaction of a daughter species $(\mathrm{H})$. Finally, it is not yet excluded that $\mathrm{HNC}$ could be stable in ice at very low temperature; but observations limit the HNC fraction from the nucleus to $6 \%$ of $\mathrm{HCN}$.

\subsection{The ${ }^{15} N$ Problem and the Origin of $C N$}

A ${ }^{14} \mathrm{~N} /{ }^{15} \mathrm{~N}$ isotopic ratio of $140( \pm 30)$ in the $\mathrm{CN}$ radical has been systematically derived (Arpigny et al. 2003; Manfroid et al. 2005) from visible observations of Hale-Bopp and other comets, including the Deep Impact target 9P/Tempel 1 (Meech et al. 2005); this is half the solar value $(270)$ and contrasts with the ${ }^{14} \mathrm{~N} /{ }^{15} \mathrm{~N}$ ratio in $\mathrm{HCN}$ from radio observations (323 \pm 46 Jewitt et al. 1997; $330 \pm 98$ Ziurys et al. 1997). Other isotopic abundances in cometary volatiles are, within the (still large) error bars, also close to solar: ${ }^{13} \mathrm{C},{ }^{18} \mathrm{O},{ }^{34} \mathrm{~S}$ (see, e.g., Table 3 of Bockelée-Morvan et al. 2004, and Altwegg and Bockelée-Morvan 2003). In comet Halley, strongly non-solar ${ }^{12} \mathrm{C} /{ }^{13} \mathrm{C}$ values were found in a limited number of grains, indicating their presolar nature; however, on the average, isotopic ratios in the grains appeared to be solar; the low ${ }^{12} \mathrm{C} /{ }^{13} \mathrm{C}$ ratio in $\mathrm{HCN}$ measured in comet C/1996 B2 (Hyakutake) by Lis et al. (1997) is likely due to contamination by another line. $\mathrm{C}^{14} \mathrm{~N} / \mathrm{C}^{15} \mathrm{~N}$ is up to now the only clearly non-solar isotopic ratio derived on the scale of the whole comet and involving heavy atom isotopes.

That $\mathrm{HCN}$ is not the unique parent of $\mathrm{CN}$ has also been questioned on other grounds: comparing $\mathrm{HCN}$ and $\mathrm{CN}$ parent scalelengths, $\mathrm{HCN}$ and $\mathrm{CN}$ production rates, or $\mathrm{CN}$ jet morphology (e.g., Fray et al. 2005). This has lead to the consideration of other parents: $\mathrm{HC}_{3} \mathrm{~N}, \mathrm{C}_{2} \mathrm{~N}_{2}$ or refractory nitrogenated compounds (Fray et al. 2004). The production of some CN from ${ }^{15} \mathrm{~N}$ rich grains is not sufficient to reconcile the ${ }^{14} \mathrm{~N} /{ }^{15} \mathrm{~N}$ measurements in $\mathrm{CN}$ with the HCN data; there is currently no proposed explanation which works.

\subsection{Detection of Carbon Disulfide $C S_{2}$}

Since the detection of the daughter species CS in comet C/1975 V1 West (Smith et al. 1980), its parent has been sought, and various candidate parent molecules have been proposed and discussed based on photodissociation lifetime arguments. That of $\mathrm{CS}_{2}$, proposed as a CS parent as early as 1982 by Jackson et al., has been revised in the laboratory from 100 to $500 \mathrm{~s}$ (at $1 \mathrm{AU}$ ), whereas cometary data required about $1000 \mathrm{~s}$ (Feldman et al. 1999). The establishment of $\mathrm{S}+\mathrm{CO}$ as main photodissociation path for OCS (Huebner et al. 1992) ruled it out as a candidate. This exemplifies the crucial role played by laboratory data in the interpretation of cometary observations. $\mathrm{CS}_{2}$ seems now to have been identified with confidence in $122 \mathrm{P} /$ de Vico, through 28 visible lines (Jackson et al. 2004). Laboratory experiments (in supersonic molecular beams) are in progress to confirm that the line intensities are correct. $\mathrm{CS}_{2}$ would be the third parent molecule (along with $\mathrm{CO}$ and $\mathrm{S}_{2}$ ) identified directly from $\mathrm{UV} /$ visible spectra.

\subsection{Polycyclic Aromatic Hydrocarbons (PAHs)}

The detection of PAHs, a major component of IS matter, would be an important link between the ISM and comets. The $3.28 \mu \mathrm{m}$ band of PAHs was seen in some comets, 
but not in Hale-Bopp, perhaps due to the large heliocentric distance (2.7 AU) where it was searched for. This band is subject to blending with $\mathrm{OH}$ prompt emission and $\mathrm{CH}_{4}$ fluorescence emission. In comet Halley, a recent reanalysis of TKS data (Clairemidi et al. 2004) added a tentative detection of pyrene $\mathrm{C}_{16} \mathrm{H}_{10}$ to that of phenanthrene $\mathrm{C}_{14} \mathrm{H}_{10}$ (Moreels et al. 1993). PAHs are seen in laser ablation of those IDPs thought to be cometary in origin (Clemett et al. 1993). Recently, PAH features have been seen in the IR spectrum of 9P/Tempel 1 after the impact of Deep Impact (Lisse et al. 2005). According to Kress et al. (2004), PAHs are not likely to be produced by the impact itself, as their formation, while thermodynamically allowed, is not favored kinetically. The search for PAHs in the cometary dust sample collected by Stardust will be extremely interesting (if they have survived the collection process).

\subsection{The $\mathrm{H}_{2} \mathrm{O}$ Reference}

A new development which improves the measurement of relative abundances is the direct determination of $\mathrm{H}_{2} \mathrm{O}$ production through space-based submillimeter observations. This complements the widely employed but indirect technique using $\mathrm{OH}$ maser emission (Crovisier et al. 2002), ground-based IR observations of hot bands of water (Dello Russo et al. 2004), narrow band UV OH emission (A'Hearn et al. 1995) and other techniques based on water photodissociation products (Feldman et al. 2004). SWAS (Bensch et al. 2004) and Odin (Lecacheux et al. 2003) have observed the $1_{10}-1_{01}$ submillimeter line of water in various comets. The ${ }^{18} \mathrm{O} /{ }^{16} \mathrm{O}$ ratio could even be determined with precision in comet 153P/Ikeya-Zhang from Odin data (Lecacheux et al. 2003). SWAS (Bensch, this volume), Odin (Biver et al. 2005b), and the smaller radio telescope MIRO aboard Rosetta all attempted to follow the 9P/Tempel 1 temporal evolution after Deep Impact. Remarkable features are the pre-impact natural variability, and the line profile changes seen in Odin spectra during and a few hours after impact.

\section{Comet Variation and Comet Population Homogeneity}

\subsection{Comet-to-Comet Variation}

As a "first approximation", for the sake of simplicity, and due to the lack of more complete data, comet composition was first thought of as universal. Dust-to-gas ratio variations were, however, already obvious (Rolfe \& Battrick 1987) and CN was used early on (A'Hearn et al. 1995) to distinguish comet classes. We are now, due to the increased sensitivity in radio and IR instruments, at the beginning of a statistical investigation of the comet population based on volatiles. Biver et al. (2002a, 2005a) and Crovisier et al. (2005) have now gathered data on a handful of comets, and dispersions in the abundances are given in Figure 1. They remain generally within a factor 3 of the mean, except for the highly volatile CO molecule. No clear correlation has yet been found with dynamical parameters characterizing the orbit.

\subsection{Comet Internal Heliocentric Variation}

The study of heliocentric variation of cometary abundances is an important tool to discuss processes responsible for the production of parent molecules (Biver et al. 2002b; "Christmas tree plot"). Similar evolution is expected in a group of molecules if the release of a single major species $\left(\mathrm{H}_{2} \mathrm{O}\right.$, or $\left.\mathrm{CO}\right)$ controls their outgassing; any departure from the average behavior indicates, to the contrary, the interplay of other processes. Several have been proposed: chemistry in the coma (HNC vs. HCN, cf. above), differential sublimation $\left(\mathrm{CO}\right.$ vs. $\left.\mathrm{H}_{2} \mathrm{O}\right)$ and release from grains $\left(\mathrm{H}_{2} \mathrm{CO}\right.$ and, possibly, $\left.\mathrm{CN}\right)$. Less pronounced departures seem to affect even the production rate ratio of quite similar 
species such as $\mathrm{H}_{2} \mathrm{O}$ and $\mathrm{CH}_{3} \mathrm{OH}$ (Biver et al. 2002b., 2005a), and a layered nucleus model has been proposed to explain it (Rodgers \& Charnley, this volume).

\subsection{Coma Maps}

Another important recent development is the ability to map molecules in the coma at radio wavelengths. This allows us to address the origin (nuclear or not) and the photodissociation lifetime of a species, and to reveal structures ("jets") in the coma. Large-scale maps (a few arcminutes, or a few $10^{5} \mathrm{~km}$ ) were produced first point-by-point (Biver et al. 1999; Hirota et al. 1999), but more homogeneous maps are produced by detector arrays (QUARRY at FCRAO; Lovell et al. 1999) or raster techniques (NRAO 12m; Milam et al., IAUS 231 poster $)$. High resolution interferometric maps $(\sim$ a few arcsecs or thousands of $\mathrm{km}$ ) have been obtained on the very bright comet Hale-Bopp (Blake et al. 1999 at OVRO; Veal et al. 2000 at BIMA; Henry et al. 2002 at PdBI). A specific technique has been developed to analyze PdBI observation of Hale Bopp - model fitting in the (uv) plane - which allows us to reconstruct the main CO jet (Henry 2003; Bockelée-Morvan et al. 2005a). Interferometric studies of comets are especially complex due to the time variability of the object, which limits the use of super-synthesis. In this respect ALMA, with its large instantaneous $u v$ coverage, will bring a noticeable improvement.

\subsection{Temperature History and Origin of Cometary Matter, Connection to ISM}

Comets are the most pristine bodies of the solar system, but just how pristine are they? How much information on IS matter will comets provide? Several indicators exist which should inform us on the past temperature history of comae. Within a simple evolutionary model of cometary nucleus formation, they could give us a "formation temperature" of the icy material, dating back to the protosolar nebula. However, radial mixing in the PSN (e.g., Bockelée-Morvan et al. 2002), as well as vertical mixing between mid-plane and high- $z$ layers of the protostellar disk ( $c f$. disk structures presented in this volume) may have led to a complex history, possibly different for refractories and volatiles.

Important temperature indicators are:

- The presence/absence of volatiles and supervolatiles. The observation of CO at large heliocentric distances in comet Hale-Bopp (Biver et al. 2002b) may indicate that cometary matter incorporated it at low temperature $(22 \mathrm{~K}$ for pure $\mathrm{CO}$ ice, up to several tens of $\mathrm{K}$ for $\mathrm{CO}-\mathrm{H}_{2} \mathrm{O}$ mixture - the exact limit is still debated, and depends on the structure of the ice: amorphous, crystalline, clathrate). It may also indicate that the nucleus internal temperature remained at such low temperatures for $4.5 \mathrm{Gyr}$, and may provide a constraint for models of the comet accretion process: perhaps $\mathrm{CO}$ was incorporated after the buildup of the ice-dust planetesimal, as the energy produced by the accretion process itself might have released this very volatile species.

$\mathrm{H}$ is strongly depleted in comets (FUSE observations of $\mathrm{H}_{2}$ in the coma are an indication of coma production by $\mathrm{H}_{2} \mathrm{O}$ photodissociation). Rare gases, also very volatile, are crucial probes of the temperature history, but have not yet been convincingly detected (Bockelée-Morvan et al. 2004). An interesting case is $\mathrm{N}_{2}$. Its detection in comets through $\mathrm{N}_{2}^{+}$is ambiguous. Nitrogen as an element is depleted in comets ( $c f$. references in Iro et al. 2003), a fact explained generally by the volatility of $\mathrm{N}_{2}$. However, some estimations give a very similar volatility for $\mathrm{CO}$ and $\mathrm{N}_{2}$, even on $\mathrm{H}_{2} \mathrm{O}$ ices, which is clearly a problem; clathrates have been proposed as a solution to this puzzle (Iro et al. 2003).

- Ortho/Para (O/P) ratios. These have been summarized by Kawakita et al. (2004). A recent development is the use of a daughter species, $\mathrm{NH}_{2}$, whose study can be used to trace the ratios in $\mathrm{NH}_{3}$. The $\mathrm{O} / \mathrm{P}$ ratios generally point toward a temperature of about $30 \mathrm{~K}$, except in one case - comet C/1986 P1(Wilson). No difference is yet seen between 
the various comets despite their different dynamical histories. But the meaning of this spin temperature is unclear: Is it the formation temperature of the molecule in the gas phase? Formation on grain surfaces? Equilibration with the comet interior during the 4.5 Gyr of its existence?

- Further constraints come from the isotopic ratios ( $\mathrm{HCN} / \mathrm{DCN}$ and $\mathrm{H}_{2} \mathrm{O} / \mathrm{HDO}$; Mousis et al. 2000), the conservation of prestellar grains and from the crystalline and/or amorphous nature of silicates and water ice (still unknown).

While an overall correspondence exists between cometary and interstellar molecules found in hot cores (Bockelée-Morvan et al. 2000), definite differences exist; one of the most interesting being the suspected relatively high abundance of ethylene glycol in cometary ices. Another result still to be understood is the deuteration level (HCN/DCN $\sim 2.3 \times 10^{-3}$ and $\mathrm{D} / \mathrm{H}_{\mathrm{H}_{2} \mathrm{O}} \sim 3 \times 10^{-4}$ ), and the presence of crystalline silicates (Crovisier et al. 1998; Hanner and Bradley 2004) not observed in the ISM (but observed around Herbig AeBe stars; Malfait et al. 1998).

\section{Conclusion: What is Important for the Future}

About 30 abundances of parent species, and nearly 20 upper limits on related compounds have already been determined in at least one comet. In parallel with the increase of this list, the following questions need to be addressed with high priority in the future: (i) the homogeneity of the cometary nucleus; (ii) the homogeneity of the comet population; (iii) the nature of the organic refractories (which likely hide many complex molecules together with heterogeneous organic polymers). The resolution of all these issues would help to elucidate the origin of cometary matter, and to determine the respective roles of the interstellar matter (from the solar system parent cloud) and of processing in the Protosolar Nebula. In view of the complexity of hot cores like Orion, of the processes in the protosolar nebula, and of the possible additional complexity of cometary chemistry, the correlation previously found between interstellar and cometary abundances remains a surprising observational fact, which needs to be explained.

Chemical analysis in situ (with Rosetta) and on samples (dust samples, with Stardust, and - hopefully soon - ice samples with a future space mission) may enlarge considerably (up to 100 species ?) the chemical inventory of comets. It will also provide the means to carry out a chiral analysis, impossible to do from remote observing; this in turn will feed models of the early Earth for which comets may have been a source of prebiotic compounds. Ground-based and space-based tele-detection (e.g., the D/H ratio with Herschel/HIFI) will allow the statistical characterization of the comet population(s). With high spatial resolution (using large radio interferometers like ALMA and large optical telescopes, close-up maps from the Rosetta orbiter with e.g., MIRO or VIRTIS), we will study comet variability and heterogeneity (analyzing the respective chemical composition of jets versus more isotropic outgassing). Space observations will also provide the fundamental reference, $Q_{\mathrm{H}_{2} \mathrm{O}}$, as they have already begun to do.

The increased precision required to model comets asks for plenty of new laboratory measurements: spectroscopy (only a few unidentified lines at $\mathrm{mm}$ wavelength but more than 4000 in the UV/visible), photodissociation rates (most of them are now only educated guesses), and collision cross sections with $\mathrm{H}_{2} \mathrm{O}$. 


\section{References}

A'Hearn, M.F., Millis, R.L., Schleicher, D.G., Osip, D.J., \& Birch, P.V. 1995, Icarus 118, 223

A'Hearn, M.F., Belton, M.J.S., Delamere, W.A., et al. 2005, Science 310, 258

Altwegg, K. \& Bockelée-Morvan, D. 2003, Space Science Reviews 106, 139

Arpigny, C., Jehin, E., Manfroid, J., Hutsemékers, D., Schulz, R., Stüwe, J.A., Zucconi, J.-M., \& Ilyin, I. 2003, Science 301, 1522

Bensch, F., Bergin, E.A., Bockelée-Morvan, D., Melnick, G.J., \& Biver, N. 2004, Ap. J. 609, 1164

Binet, L., et al. 2004, in Astrobiology: Future Perspectives (ASSL Vol. 305), 333

Biver, N., et al. 1999, A. J. 118, 1850

Biver, N., et al. 2002a, Earth Moon and Planets 90, 323

Biver, N., et al. 2002b, Earth Moon and Planets 90, 5

Biver, N., et al. 2005a, $A \mathscr{E} A$, in press

Biver et al. 2005b, Asteroids, Comets and Meteors 2005 (IAU Symp. 229), abstract

Biver, N., et al. 2005c, BAAS 37, 710

Blake, G.A., Qi, C., Hogerheijde, M.R., Gurwell, M.A., \& Muhleman, D.O. 1999, Nature 398, 213

Bockelée-Morvan, D., et al. 2000, A\&\&A 353, 1101

Bockelée-Morvan, D., Gautier, D., Hersant, F., Huré, J.-M., \& Robert, F. 2002, A 6 A 384, 1107

Bockelée-Morvan, D., Crovisier, J., Mumma, M.J., \& Weaver, H.A. 2004, in Comets II (Univ. Arizona Press), 391

Bockelée-Morvan, D., et al. 2005a, A\&A in preparation

Bockelée-Morvan, et al. 2005b, Abstract, Asteroids, Comets and Meteors 2005 (IAU Symp. 229), abstract

Canaves, M.V., De Almeida, A.A., Boice, D.C., \& Sanzovo, G.C. 2002, Earth Moon and Planets 90,335

Charnley, S.B., Rodgers, S.D., Butner, H.M., \& Ehrenfreund, P. 2002, Earth Moon and Planets 90, 349

Clairemidi, J., Bréchignac, P., Moreels, G., \& Pautet, D. 2004, Plan. Sp. Sci. 52, 761

Clemett, S.J., Maechling, C.R., Zare, R.N., Swan, P.D., \& Walker, R.M. 1993, Science 262, 721

Crovisier, J. 2004, in Astrobiology: Future Perspectives (ASSL Vol. 305), 179

Crovisier, J., Colom, P., Gérard, E., Bockelée-Morvan, D., \& Bourgois, G. 2002, A $\& A$ 393, 1053

Crovisier, J., Leech, K., Bockelée-Morvan, D., Brooke, T.Y., Hanner, M.S., Altieri, B., Keller, H.U., \& Lellouch, E. 1997, Science 275, 1904

Crovisier, J., Bockelée-Morvan, D., Colom, P., Biver, N., Despois, D., Lis, D.C., \& the Team for target-of-opportunity radio observations of comets 2004a, $A \mathscr{E} A 418,1141$

Crovisier, J., Bockelée-Morvan, D., Biver, N., Colom, P., Despois, D., \& Lis, D.C. 2004b, A\&A 418, L35

Crovisier, J., et al. 2005, BAAS 37, 646

Despois, D. \& Cottin, H. 2005, in Lectures in Astrobiology Vol. I, eds. M. Gargaud, B. Barbier, H. Martin, \& J. Reisse (Springer), 289

Ehrenfreund, P., Charnley, S.B., \& Wooden, D. 2004, in Comets II (Univ. Arizona Press), 115

Fegley, B., Jr. 1993, in The Chemistry of Life's Origins, eds. Greenberg, Mendoza-Gomez and Pirronello (Kluwer), 75

Feldman, P.D., Weaver, H.A., A'Hearn, M.F., Festou, M.C., McPhate, J.B., \& Tozzi, G.-P. 1999, $B A A S 31,1127$

Feldman, P.D., Cochran, A.L., \& Combi, M.R. 2004, in Comets II (Univ. Arizona Press), 425

Festou, M.C., Keller, H.U., \& Weaver, H.A. (eds.) 2004, Comets II (Univ. Arizona Press)

Fomenkova, M.N. 1999, Space Science Reviews 90, 109

Fray, N., Bénilan, Y., Cottin, H., Gazeau, M.-C., Minard, R.D., \& Raulin, F. 2004, Meteoritics and Planetary Science 39, 581

Fray, N., Bénilan, Y., Cottin, H., Gazeau, M.-C., \& Crovisier, J. 2005, Plan. Sp. Sci. 53, 1243

Hanner, M.S. \& Bradley, J.P. 2004, in Comets II (Univ. Arizona Press), 555

Henry, F. 2003, Ph.D. Thesis, Paris

Henry, F., Bockelée-Morvan, D., Crovisier, J., \& Wink, J. 2002, Earth Moon and Planets 90, 57 
Hirota, T., Yamamoto, S., Kawaguchi, K., Sakamoto, A., \& Ukita, N. 1999, Ap. J. 520, 895

Hollis, J.M., Lovas, F.J., Jewell, P.R., \& Coudert, L.H. 2002, Ap. J. 571, L59

Huebner, W.F., Keady, J.J., \& Lyon, S.P. 1992, Astr. Sp. Sci. 195, 291

Iro, N., Gautier, D., Hersant, F., Bockelée-Morvan, D., \& Lunine, J.I. 2003, Icarus 161, 511

Irvine, W.M., et al. 1998, Nature 393, 547

Irvine, W.M., Senay, M., Lovell, A.J., Matthews, H.E., McGonagle, D., \& Meier, R. 2000, Icarus 143,412

Irvine, W.M., Schloerb, F.P., Crovisier, J., Fegley, B., \& Mumma, M.J. 2000, in Protostars and Planets IV (Univ. Arizona Press), 1159

Irvine, W.M., Bergman, P., Lowe, T.B., Matthews, H., McGonagle, D., Nummelin, A., \& Owen, T. 2003, Origins of Life and Evolution of the Biosphere 33, 609

Jackson, W.M., Halpern, J.B., Feldman, P.D., \& Rahe, J. 1982, A\& A 107, 385

Jackson, W.M., Scodinu, A., Xu, D., \& Cochran, A.L. 2004, Ap. J. 607, L139

Jewitt D.C., Matthews, H.E., Owen, T., \& Meier, R. 1997, Science 278, 90

Kawakita, H., Watanabe, J.-I., Furusho, R., Fuse, T., Capria, M.T., De Sanctis, M.C., \& Cremonese, G. 2004, Ap. J., 601, 1152

Keller, H.U., Jorda, L., Kuppers, M., et al. 2005, Science 310, 281

Kress, M., Tielens, A., \& Frenklach, M. 2004, 35th COSPAR Scientific Assembly, 3692

Lecacheux, A., et al. 2003, A\&SA 402, L55

Lis, D.C., et al. 1997, Icarus 130, 355

Lisse, C.M., van Cleve, J., Fernandez, Y.R., \& Meech, K.J. 2005, IAU Circ. 8571, 2

Lovell, A.J. 1999, Ph.D. Thesis,

Magee-Sauer, K., Dello Russo, N., DiSanti, M.A., Gibb, E., \& Mumma, M.J. 2002, BAAS 34, 868

Malfait, K., Waelkens, C., Waters, L.B.F.M., Vandenbussche, B., Huygen, E., \& de Graauw, M.S. 1998, $A \& 3 A$ 332, L25

Manfroid, J., Jehin, E., Hutsemékers, D., Cochran, A., Zucconi, J.-M., Arpigny, C., Schulz, R., \& Stüwe, J.A. 2005, A\&SA 432, L5

Meech, K.J., Ageorges, N., A'Hearn, M.F., et al. 2005, Science 310, 265

Moreels, G., Clairemidi, J., Hermine, P., Brechignac, P., \& Rousselot, P. 1994, A\&A 282, 643

Mousis, O., Gautier, D., Bockelée-Morvan, D., Robert, F., Dubrulle, B., \& Drouart, A. 2000, Icarus 148,513

Rodgers, S.D. \& Charnley, S.B. 2001, MNRAS 323, 84

Rolfe, E.J. \& Battrick, B. 1987, Diversity and Similarity of Comets (ESA SP-278)

Smith, A.M., Stecher, T.P., \& Casswell, L. 1980, Ap. J. 242, 402

Veal, J.M., et al. 2000, A. J. 119, 1498

Ziurys, L.M., Savage, C., Brewster, M.A., Apponi, A.J., Pesch, T.C., \& Wyckoff, S. 1999, Ap. J. 527, L67 